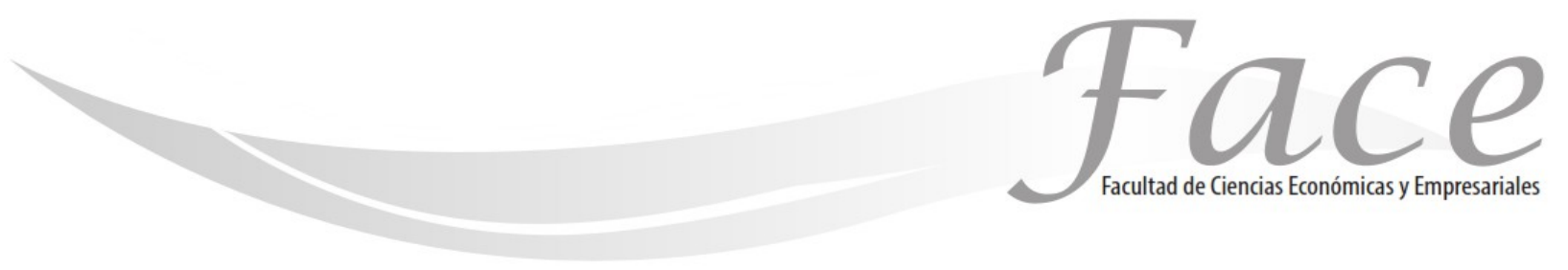

ISSN Impreso: 1794-9920

ISSN Electrónico: 2500-9338

Volumen $17-\mathrm{N}^{\circ} 2$

Año 2017

Págs. 91-99

\title{
CARACTERIZACIÓN SECTORIAL Y LABORAL DE LAS COOPERATIVAS EN EL MUNICIPIO DE PAMPLONA
}

\author{
Nathalie Hernández Pérez * \\ Enlace ORCID: http: 0000-0002-7390-9261 \\ Gendler Alexander Jaimes Gauta ** \\ Enlace ORCID: http: 0000-0001-5164-3872 \\ Jemay Mosquera Téllez *** \\ Enlace ORCID: http:// 0000-0001-5989-5644.
}

Fecha de Recepción: 15 diciembre 2016

Fecha de Aprobación: 10 marzo 2017

\section{Resumen:}

El sector solidario en la economía se ha caracterizado por fortalecer las capacidades individuales a partir de la organización de distintos actores con intereses similares. Sin embargo, en el ámbito local, no siempre existen datos sobre las dinámicas de dicho sector. En particular, el municipio de Pamplona no cuenta con análisis actualizado del sector cooperativo que permita justificar la importancia del sector solidario y su relación con las políticas de generación de empleo y reducción de la informalidad. Por lo tanto, en el presente documento se estudia la forma como las determinantes geográficas, culturales, sociales y económicas locales influyen en el comportamiento de la economía solidaria de Pamplona, se identifica la composición del sector solidario y se establecen las características del sector cooperativo y su incidencia en el desarrollo municipal en cuanto a generación de ingresos y aumento de consumo de bienes y servicios. Los resultados obtenidos permiten sentar las bases para la definición de estrategias tendientes a fortalecer el papel de las cooperativas en la generación de empleo, el sostenimiento colectivo y el crecimiento económico regional.

Palabras Claves: asociación, empleo, economía solidaria, sector solidario. 
* Economista, Estudiante de Maestría en Paz, Desarrollo y Resolución de Conflictos, Docente y Miembro del Grupo de Investigación Ciencias Económicas y Empresariales GICEE de la Universidad de Pamplona. Consultora en temas de desarrollo económico territorial nahepe@hotmail.com

"Economista, Especialista en Gerencia Financiera, Estudiante de Maestría en Ciencias Económicas y Empresariales. Consultor en temas de desarrollo económico local gendler_85@hotmail.com

${ }^{* * \star}$ Arquitecto, doctor en Arquitectura y Posdoctor en Ciudades y Megalópolis. Director del Grupó de Investigación Gestión Integral del Territorio - GIT de la Universidad de Pamplona. Consultor en temas de ordenamiento territorial y desarrollo urbano integrado jemay.mosquera@hotmail.com

\title{
SECTORAL AND LABOR CHARACTERIZATION OF COOPERATIVES IN THE MUNICIPALITY OF PAMPLONA
}

\begin{abstract}
The solidarity sector in the economy has been characterized by the strengthening of individual capacities from the organization of different actors with similar interests. However, data on the dynamics of this sector do not always exist at the local level. In particular, the municipality of Pamplona does not have an updated analysis of the cooperative sector that would justify the importance of the solidarity sector and its relationship with employment generation and informality reduction policies. Therefore, the present paper examines how local geographical, cultural, social and economic determinants influence the behavior of the solidarity economy of Pamplona, identifies the composition of the solidarity sector and establishes the characteristics of the cooperative sector and its impact on municipal development in terms of income generation and increased consumption of goods and services. The obtained results provide the basis for the definition of strategies aimed at strengthening the role of cooperatives in employment generation, collective support and regional economic growth.

Keywords: association, employment, solidarity economy, solidarity sector.
\end{abstract}

\section{CARACTERIZAÇÃO E SETOR TRABALHO COOPERATIVO NO MUNICÍPIO DE PAMPLONA}

\begin{abstract}
Resumo
O setor de solidariedade na economia tem sido caracterizada por reforçar as capacidades individuais de organizar vários actores com interesses semelhantes. No entanto, a nível local, eles nem sempre existem dados sobre a dinâmica do setor. Em particular, o município de Pamplona não actualiza o sector cooperativo justificando a importância do sector da solidariedade e sua relação com as políticas de geração de emprego e redução da análise informalidade. Portanto, aqui como determinantes econômicos geográficas, culturais, sociais e locais influenciam o comportamento da economia solidária Pamplona é estudado, a composição do sector da solidariedade é identificado e as características do sector cooperativo e estabelece seu impacto no desenvolvimento municipal em termos de geração de renda e aumento do consumo de bens e serviços. Os resultados obtidos fornecem a base para a definição de estratégias para fortalecer o papel das cooperativas na criação de emprego, 0 apoio coletivo e crescimento econômico regional.
\end{abstract}

Palavras-chave TIC, da Competitividade, Educação, habilidades cognitivas, comunas. 


\section{INTRODUCCIÓN:}

En el presente artículo, se realiza un análisis de las cooperativas en el municipio de Pamplona, las cuales se encuentran dentro del sector solidario, en tanto hacen parte de las organizaciones que tienen principios y valores como la ayuda mutua, la democracia, la solidaridad, la responsabilidad, la igualdad y la equidad. Dichos principios, les otorgan un carácter social en la medida que su base fundamental es el capital social relacionado con las organizaciones sociales y como estas logran la cooperación para el beneficio mutuo a partir de redes, normas y confianza.

El desarrollo de la caracterización de las cooperativas fue llevado a cabo en el año 2016 y se fundamentó en autores que resaltan los beneficios del capital social (Putman, año; Azuero Rodríguez, 2009 y Klisberg 2009 y 2017), no aplica poner página porque no es una cita textual los cuales sostienen que la economía del tercer sector se ha visto como una opción alterna a muchas deficiencias que tiene el sistema económico actual y que puede sobrevivir en él, ya que este sector contiene las características relevantes para disminuir la pobreza y la desigualdad en los diferentes países, mejorando las condiciones de los trabajadores. Así las cosas, el tercer sector o sector social incluye todas las organizaciones sin ánimo de lucro como: fundaciones, asociaciones mutuales, fondos de empleados, corporaciones, organizaciones no gubernamentales y cooperativas.

\section{METODOLOGÍA:}

Para cumplir con los objetivos planteados, se realizó una investigación cuantitativa utilizando las herramientas que ofrece la estadística descriptiva. En primer lugar, se llevó a cabo una revisión de la literatura relacionada con el sector solidario y el cooperativismo en el ámbito internacional, nacional y regional, con el fin de conocer e interpretar las dinámicas del cooperativismo desde diferentes perspectivas y escenarios geográficos. Posteriormente, se desarrolló la etapa cuantitativa a partir de fuentes secundarias y primarias. En el primer caso, se tomó la información de las cooperativas registradas en Cámara de Comercio del municipio de Pamplona en el año 2016, lo que permitió clasificarlas por actividad. En el segundo caso, se realizó una investigación no experimental de corte transversal, en la cual se hizo uso de la estadística descriptiva para mostrar las principales características de las cooperativas en el municipio. Por último, para la recopilación y análisis de información o datos, se aplicó una encuesta con preguntas cerradas, que contempló características correspondientes al sector económico, generación de empleo, seguridad social e interés de asociatividad.

\section{RESULTADOS Y DISCUSION:}

\section{COOPERATIVAS: GENERALIDADES $Y$ ASPECTOS BÁSICOS}

En América Latina, (=) a partir de la última década del siglo pasado y gracias a procesos de democratización, descentralización y modernización del Estado, se ha venido reforzando la legitimidad e importancia de los gobiernos municipales y el papel protagónico de las ciudades; la apertura ha movilizado agentes económicos que saben la necesidad de los gobiernos locales por crear condiciones de competitividad y productividad. (Zuluaga, Mosquera, Gómez y Peñalosa, 2012). Al mismo tiempo, el desarrollo exige priorizar la creación de satisfactores endógenos y sinérgicos, asumir simultáneamente las carencias como potencialidades y reconocer relaciones de auto-dependencia desde la base de la sociedad para lograr el empoderamiento local y obtener mayores efectos sinérgicos en el territorio (Max-Neef, Elizalde y Hopenhayn, 1986). (=)

Desde esta perspectiva, adquiere relevancia el enfoque sistémico del territorio que promueve el Departamento Nacional de Planeación (DNP) y que se soporta diferentes instancias del orden nacional para reconocer la importancia de las condiciones biofísicas, tecnológicas, sociales, económicas y políticas del entorno y de la identificación de sus múltiples interacciones. (IGAC, 1997). Por su lado, el sistema económico está compuesto por tres subsistemas. El primero corresponde al sector privado, el segundo al sector público y el tercero es conocido como el sector solidario, que hace parte de la economía social.

Klisberg (1999) destaca que la crisis mundial en parte responde a un problema de valores de la sociedad, como es el caso de falta de ética en la economía convencional. El mismo autor menciona que la insolidaridad es la causa de una sociedad injusta y poco valorativa por el bienestar del otro, de donde la humanidad pierde valores, ética y respeto por la vida misma, donde la responsabilidad social precipita la crisis económica y social. Al respecto, propone consolidar esfuerzos encaminados a resolver los grandes desafíos del mundo contemporáneo y lograr las metas de desarrollo sostenible de las Naciones Unidades, reivindicando el papel de la justicia y de los organismos encargados de impartirla en la generación y aplicación de políticas sociales justas y equitativas para lograr sociedades responsables desde la convivencia, el respeto y el aprecio mutuo. (Klisberg, 2017) 
Por otra parte, el capital social incrementa los beneficios cuando se invierte en capital físico y capital humano, de tal forma que al fortalecer el capital social mejora la rentabilidad de la inversión. (Azuero Rodríguez, 2009).

Las cooperativas hacen parte del sector solidario y se pueden clasificar de acuerdo a la forma como desarrollan sus actividades u objeto en: especializadas, multiactivas e integrales. Las especializadas como su nombre lo indica, desarrollan una sola actividad que puede estar en cualquier sector económico como producción, comercialización, financiamiento 0 consumo; por otra parte, las multiactivas se especializan en dos 0 más actividades que no necesariamente están relacionadas como es el prestar servicios de salud y educación o producción agrícola y crédito; finalmente, las cooperativas integrales se caracterizan porque cumplen con todo el ciclo económico, producen, comercializan y financian.

Los emprendimientos cooperativos en América Latina iniciaron en Argentina, Brasil, México y Venezuela a mediados siglo XIX, las actividades que predominaron fueron consumo y financiamiento. Varias causas influyeron en la conformación de estas organizaciones como: inmigrantes europeos, la iglesia, los gobiernos y los sindicatos; independientemente de las razones por las que se haya implementado en los diferentes países de América Latina, el impacto de estas organizaciones en lo económico y social ha sido fuerte, especialmente en la generación de empleos como es el caso de Bolivia en donde las cooperativas mineras emplean aproximadamente 100.000 personas, por tal motivo, la Organización Internacional del Trabajo (OIT) apoyan la creación de cooperativas como estrategia de lucha contra la pobreza. (Mogrovejo, Mora \& Vanhuynegem, 2012, p.13-14).

El carácter especial de las organizaciones, según Hernández \& Cruz (2014), permite a los asociados controlar de forma democrática su destino en la búsqueda del beneficio mutuo y de la responsabilidad, mejoran sus condiciones de vida logrando la igualdad, la equidad y la solidaridad.

\section{Dadas estas particularidades:}

"El cooperativismo en Colombia representa un importante agente para el desarrollo socio empresarial y el fortalecimiento del capital social, desarrollando actividades de la más diversa índole, fortaleciendo en ocasiones al mercado y en otras solucionando fallas de éste y del Estado" (Álvarez y Serrano, 2006, p.3).

El cooperativismo en Colombia aparece en los años treinta, en los primeros cuarenta años de existencia entre (19311971), el cooperativismo en Colombia es muy escaso, sus representantes carecen de vida propia, aunque se vale resaltar que el movimiento se desenvolvió con bastante propiedad y siempre mantuvo sus postulados básicos. Las cooperativas más antiguas de Colombia fueron creadas entre los años de 1932 y 1945, estas se encuentran ubicadas en los departamentos de Antioquia, Valle del cauca, Santander, Caldas, Huila y Tolima. (Confecoop, 2015).

Durante la década de los años 80 del siglo pasado, el cooperativismo vivió su gran crisis debido a problemas ideológicos y de doctrina además del síndrome burocrático dentro del cooperativismo y la falta de credibilidad de la opinión pública y del Estado. En este periodo, se creyó que el cooperativismo en Colombia había llegado a su etapa de madurez y era la hora de extraer de la crisis lo mejor de las experiencias obtenidas y así lograr nuevas proyecciones para el sector.

De acuerdo con lo expuesto, en el periodo comprendido entre los años 1991 y 2001, existe una transformación del cooperativismo hacia la economía solidaria, jalonada también por los grandes cambios vividos en el país, entre los que se destacaron la apertura económica, el neoliberalismo y la globalización, que llevaron a que los nacionales empezaran a consumir productos extranjeros y cayera la demanda y la producción de bienes nacionales.

Posteriormente, con la ley 454 de 1998, el Departamento Administrativo Nacional de Cooperativas (DANCOOP), se transformó en el Departamento Administrativo Nacional de Economía Solidaria (DANSOCIAL), el cuál lideraría las riendas y la estructuración del tercer sector, como se le denomina también a la Economía Solidaria en los próximos años. Finalmente, DANSOCIAL, mediante el decreto 4122 de 2011, fue reemplazada por la Unidad Administrativa Especial de Organizaciones Solidarias, instancia que coordina y lidera los procesos de la Economía Solidaria en la actualidad.

Según la Superintendencia de Economía Solidaria (2013), en Colombia, a pesar de que en más del $80 \%$ de los municipios existen cooperativas, hay una concentración de aproximadamente un $60 \%$ de las cooperativas en los departamentos donde se encuentran las cuatro principales ciudades del país: Bogotá, Medellín, Cali y Bucaramanga. Por otra parte, el $56 \%$ de las cooperativas son microempresas, el $33 \%$ son pequeñas empresas, el $8 \%$ son medianas empresas y el $3 \%$ son grandes empresas.

La estructuración de las cooperativas parte de las nociones de solidaridad, libre adhesión y empresarialidad asociativa que se encuentran en la Constitución Política y que están reglamentadas con la Ley 79 de 1998. Estas normas, también amparan otras formas asociativas como los fondos de empleados, entendidos como una figura en la que los trabajadores de una misma entidad desarrollan actividades 
Nathalie Hernández Pérez - Gendler Alexander Jaimes Gauta- Jemay Mosquera Téllez

de ahorro y crédito para sus propias necesidades; y también las asociaciones mutuales, las cuales son formas asociativas con interés mutuo en la que los inversores destinan los excedentes de su actividad a una causa determinada y no son beneficiarios de su actividad.

Posteriormente, para ampliar el marco del sector cooperativo, se desarrolló la Ley 454 de 1998, la cual incluye a cooperativas, empresas comunitarias y asociaciones empresariales que realicen actividades sin ánimo de lucro y que satisfagan las necesidades de sus asociados. Dentro de esta ley, se encuentran los principios y fines de la economía solidaria como son: gestión democrática de la empresa y hacer uso de sus excedentes para prestar servicios a sus asociados.

Como se mencionó anteriormente, mediante el decreto número 4122 del 2011, el fomento de la estructuración del tercer sector quedó a cargo de la Unidad Administrativa Especial de Organizaciones Solidarias, la cual es la responsable de la coordinación de las políticas públicas de promoción, planeación, protección, fortalecimiento y desarrollo empresarial de las organizaciones de economía solidaria. Desde esta Unidad, se ha venido estructurando un tercer sector conformado por empresas cooperativas mutuales y de interés general, con un solo objetivo que apunta a la solidaridad como integradora de Organizaciones No Gubernamentales (ONG) y cualquier organización sin ánimo de lucro.

Tabla 1. Estructura organizacional de la Economía Solidaria en Colombia

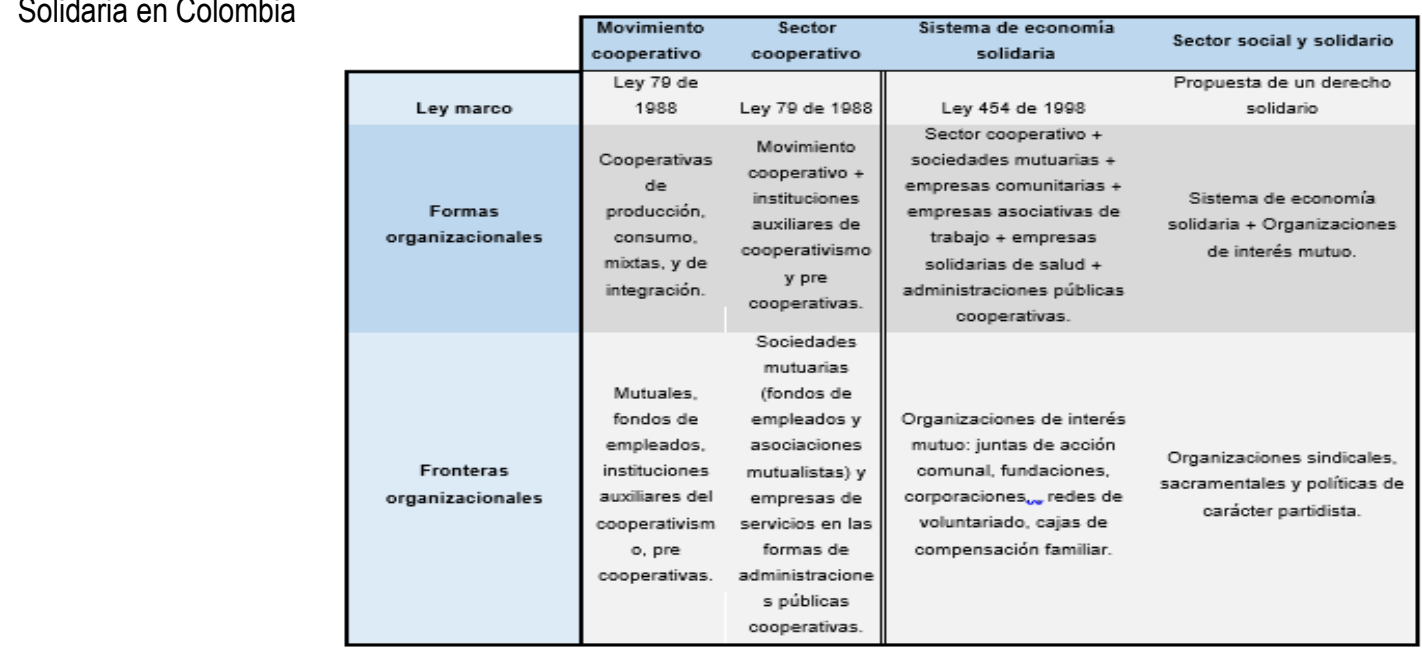

Fuente: Elaboración propia, a partir de Ley 79 de 1988 y

Ley 454 de 1998
Esta integración que se propone conformar se denomina sector ampliado de la economía solidaria o sector social y solidario. Con dicha propuesta el cooperativismo seguiría jugando un papel importante referente a la conformación de empresas mutuales.

Por su lado, la Confederación de Cooperativas de Colombia (CONFECOOP), es el organismo de integración gremial de grado superior en Colombia y su función consiste en unificar la representación y defensa del sector cooperativo colombiano en el entorno nacional e internacional. Además, Confecoop se encarga de recolectar la información de las distintas empresas solidarias junto con a la Superintendencia de la Economía Solidaria.

Adicionalmente, existe un organismo de segundo grado que es la Asociación Colombiana de Cooperativas (ASCOOP), que tiene como principal función defender la identidad cooperativa de fomento, promoción y divulgación. Entre sus principales actividades se destacan, la educación dentro y fuera de las cooperativas y la prestación de servicios de auditoría, la revisoría fiscal y el acompañamiento a la constitución de empresas cooperativas y el mejoramiento de sus operaciones.

La estructura organizacional del cooperativismo y la economía solidaria en Colombia se muestra en la siguiente tabla sobre la base de tres variables: las leyes que dan vigencia a uno u otro contenido conceptual; las formas organizacionales contenidas en Ley o propuestas; y las fronteras existentes con organizaciones aparentemente pertenecientes a uno u otro contenido conceptual. 


\section{COOPERATIVAS DE PAMPLONA: CARACTERÍSTICAS Y DINÁMICAS}

Para la realización del estudio, se clasifico el sector cooperativo del municipio de Pamplona a partir de la base de datos de registro mercantil y la visita a las cooperativas activas, pues a pesar de las ventajas que se encuentran en este tipo de organización, solo el $11 \%$ de ellas han renovado su matrícula mercantil pero las que permanecen están establecidas hace más de 20 años indicando que son organizaciones sostenibles.

Según la Cámara de Comercio de Pamplona, en el municipio existen formalmente 228 organizaciones sin ánimo de lucro, de las cuales 44 han sido registradas como cooperativas. Además, resalta el hecho que para el 2016 solo se encuentran activas 5 cooperativas. El tipo de organización que tiene mayor participación es la asociación con un $49 \%$, seguido por las cooperativas con el $19,29 \%$ y de las fundaciones con un 33\% como se puede ver en la ilustración 1.

Ilustración 1. Clasificación de las organizaciones del municipio de Pamplona

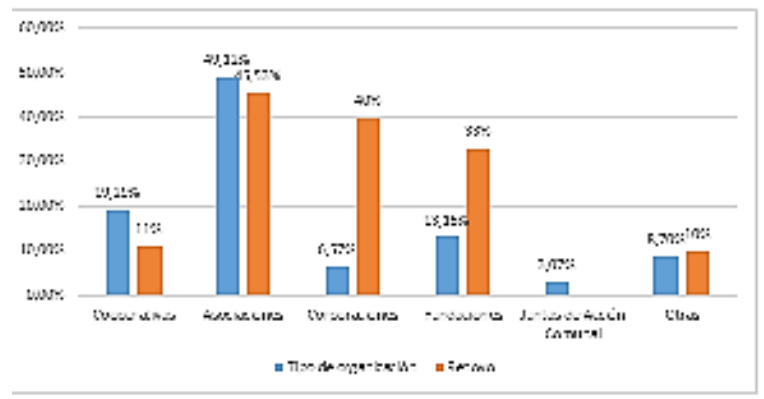

Fuente: Elaboración propia a partir de Cámara de Comercio

Las asociaciones, además de ser las que tienen mayor participación, también son las organizaciones que han tenido mayor sostenibilidad, pues el $45,53 \%$ han renovado su registro seguidas por las corporaciones con el $40 \%$ de renovaciones y las fundaciones con el $33 \%$. Por el contrario, las Juntas de Acción Comunal se conformaron entre los años de 1996 y 1997 pero ninguna renovó su registro.

Como se pudo evidenciar, la creación de cooperativas ha tenido tendencias en diferentes periodos, mostrando un auge en 1996 y nuevamente en el año 2012, tal y como lo indica la siguiente ilustración. Es importante aclarar que la base de datos registra la información desde 1996, sin embargo, de acuerdo con la información primaria obtenida, la empresa más antigua tiene 53 años de haber sido creada.
Ilustración 2. Creación de Cooperativas en el municipio de Pamplona

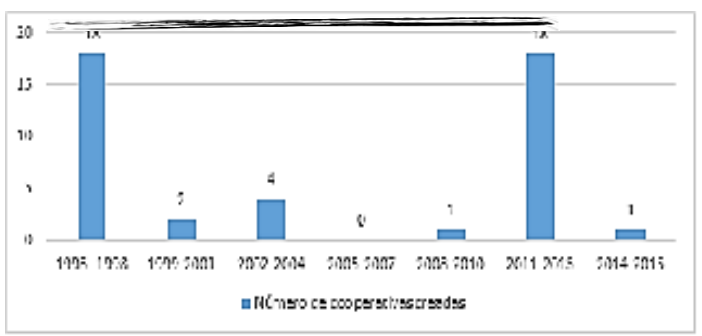

Fuente: Elaboración propia a partir de Cámara de Comercio Pamplona

Según las entrevistas realizadas a las cooperativas del municipio, la principal razón para conformar la cooperativa fue la unión del talento humano y la unión de capitales. Un ejemplo de esto, es la Cooperativa de Transportadores el Motilón "COOPMOTILON", la cual en su historia de formación cuenta que surgió de la necesidad de:

"...protestar contra los desmanes administrativos con que los venían tratando, sin tener en cuenta sus condiciones. Poco a poco la empresa los estaba presionando y llevando a perder las pocas oportunidades de trabajo, desesperándolos ya que este era el único sustento de su familia. Inquietos por la situación se fue gestando un grupo de personas que sabían que someterse no era la solución, la solución era unirse para crear una empresa que los tomara en cuenta como personas, que les brindará oportunidades, pero sobre todo igualdad para poder laborar dignamente y darles a sus familias un gran ejemplo de superación, es así como empieza a gestarse la cooperativa."

Pese a que, desde el año 1996 hasta el año 2015, se crearon 44 cooperativas, solo 5 renovaron su registro después del año de creación. A pesar de que oficialmente renovó solo el $11 \%$, a partir de la información primaria se detectó que existe una cooperativa dedicada al reciclaje que continúa trabajando, pero se encuentra sin recursos suficientes para hacer su actualización.

Ilustración 3. Renovación de registro mercantil

Fuente: Elaboración propia a partir de Cámara de Comercio Pamplona

El estudio demostró que Las cooperativas especializadas, es decir, las que se dedican a una sola actividad tienen mayor número de matrículas, pues en el año 2012 todas 
Nathalie Hernández Pérez - Gendler Alexander Jaimes Gauta- Jemay Mosquera Téllez

las registradas lo hicieron bajo esta modalidad de desarrollo de actividades; sin embargo, ninguna de ellas existe en la actualidad, posiblemente porque todas fueron cooperativas de trabajo asociado y, dadas las directrices del Ministerio de Trabajo formuladas en el mismo año 2012, las cooperativas que no cumplieran con los requisitos de vinculación y pago de prestaciones sociales como asalariados debían entrar en liquidación. Según funcionarios de la Cámara de Comercio de Pamplona, en la actualidad si se encuentran registradas cooperativas de trabajo en esta oficina, pero llevan a cabo sus actividades en municipios cercanos que no tienen Cámara de Comercio y por ello se registran en la de Pamplona.

Ilustración 4. Clasificación por desarrollo de actividades

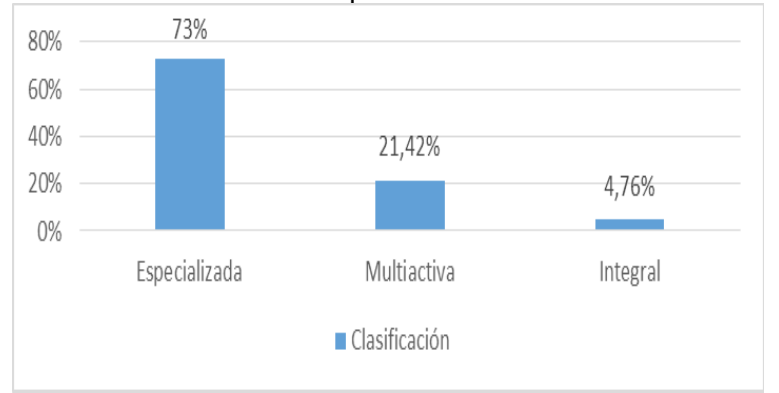

Fuente: Elaboración propia a partir de Cámara de Comercio

En lo que respecta a sus operaciones, se observa que las cooperativas activas llevan a cabo sus actividades en el sector terciario y prestan servicios de transporte, comercialización y financieros. Además, a pesar que las cooperativas de transporte iniciaron como especializadas, estas han diversificado sus servicios ampliando sus actividades al sector de hotelería y alimentación.

En cuanto al tiempo de actividad, dentro de las cooperativas con mayor antigüedad se encuentra la Cooperativa Multiactiva de Empleados Trabajadores y Particulares de la República de Colombia (COOMULTRUP), la cual se fundó hace 39 años y es integral, en tanto se dedica a comercializar, distribuir y realizar créditos. Esta cooperativa es una de las más antiguas; sin embargo, la más antigua se estableció hace 53 años como Cooperativa de Transportadores Nacionales de Pamplona Ltda (COTRANAL), seguida por la Cooperativa de Transportadores el Motilón "COOPMOTILON", fundada hace 23 años.

Por otra parte, es necesario tener en cuenta a la Cooperativa Multiactiva de Trabajadores de Santander

"COOMULTRASAN", organización fundada hace 53 años, pero establecida en el Municipio hace 13 años, la cual ofrece productos para el hogar, materiales para construcción, servicios del Plan Obligatorio de Salud-POS, servicios de salud complementarios, educación y capacitación.

A pesar de que pocas cooperativas permanecen en el Municipio, estas son fuentes de empleos directos e indirectos, pues generan 42 empleos directos y 147 empleos indirectos. Los empleados directos desarrollan actividades como auxiliares profesionales, secretaría y gerencia, por tanto, reciben un salario y se encuentran afiliados a seguridad social. Cabe destacar, que los 42 empleos son generados por 4 empresas, pues solamente hay una que no cuenta con personas dedicadas a la administración.

En lo concerniente a los empleos indirectos, estos son generados principalmente por las dos cooperativas dedicadas al servicio de transporte y la cooperativa de reciclaje, que cuenta con 35 asociados. Estos asociados son independientes y, excepto los que se dedican a la actividad de reciclaje, cuentan con afiliación a la seguridad social. Teniendo en cuenta que los asociados a la Cooperativa de reciclaje no pagan afiliación y de acuerdo con lo expresado por un asociado, estos se afilian a los programas que tiene el Estado y entre los asociados recolectan, de forma voluntaria, cuotas para las personas de mayor edad que ya no laboran debido a que no cotizaron pensión.

\section{CONCLUSIONES}

El sector solidario internacionalmente ha tenido relevancia histórica, especialmente en la reivindicación de los derechos de los trabajadores y, laboralmente, acoge un porcentaje notable de la población; sin embargo, solo hasta los últimos años se ha soportado teóricamente en el capital social.

En América Latina y en Colombia, las organizaciones del sector solidario se encuentran claramente reguladas y cuentan con la legislación y las instituciones que generan el acompañamiento y control para que pueda operar. No obstante, se evidencia la ausencia de estudios recientes sobre estas organizaciones, especialmente sobre las que están enfocadas al cooperativismo.

El estudio permitió identificar que, dentro del sector solidario existente en el municipio de Pamplona, predominan las asociaciones por encima de las cooperativas. Sin embargo, el sector cooperativo presente 
genera desarrollo local, pues además de ser una fuente de empleos directos e indirectos, sus características y dinámicas aportan al mejoramiento del bienestar de sus asociados y prestan servicios que contribuyen con la calidad de vida de los habitantes del municipio. Por otra parte, la antigüedad demuestra que son organizaciones sostenibles que generan mayores ingresos y a su vez aumentan el consumo de bienes y servicios, lo que conlleva a una mayor dinámica económica.

Dentro de las características que permiten explicar la sostenibilidad de estas organizaciones, se encontró la capacidad de adaptarse a los cambios y a las nuevas necesidades de la población, la cual es evidente en la diversificación de la oferta de servicios tendientes a satisfacer a los clientes en sus nuevos requerimientos.

Así como las cooperativas que se mantienen activas ante la Cámara de Comercio de Pamplona, cumplen con los principios y la reglamentación de las organizaciones de este sector, la que no se encuentra al día no garantiza los principios del cooperativismo ni asigna presupuesto para la capacitación de sus asociados. Teniendo en cuenta esta problemática, vale la pena generar acompañamiento a esta cooperativa que cuenta con 35 asociados dado que ellos ya han identificado la importancia que tiene el capital social en su bienestar, pero requieren programas que mejoren el desarrollo de su organización.

Por último, se observa la necesidad latente de identificar las fortalezas y mejorar los lazos de confianza y cooperación presentes en el municipio de Pamplona, para lograr configurar nuevas organizaciones y consolidar las cooperativas existentes, de tal manera que su contribución sea más efectiva en la reducción de las brechas sociales y condiciones de pobreza de las comunidades vulnerables. Es necesario también, promover proyectos productivos que permitan el desarrollo de capacidades organizaciones y comunitarias y que, al igual que el transporte, respondan a necesidades sentidas de la población, como prerrequisitos para asegurar la sostenibilidad de las cooperativas y las organizaciones del sector solidario en el tiempo.

\section{REFERENCIAS:}


ISBN: 978-987-4057-70-9. Editorial Jusbaires. Buenos Aires. $140 \mathrm{p}$.

- Max Neef, M.; Elizalde, A. y Hopenhayn, M. (1986). Desarrollo a Escala Humana -Una Opción para el Futuro-CEPAUR. Santiago de Chile. p. 96

- Mogrovejo, R.; Mora, A. \& Vanhuynegem, P., Eds. (2012). El cooperativismo en América Latina. Una diversidad de contribuciones al desarrollo sostenible. La Paz, OIT, Oficina de la OIT para los Países Andinos, 2012. 400 p. ISBN: 978-92-2-326393-5

- Organizaciones Solidarias. http://www.orgsolidarias.gov.co/

- Zuluaga L, Mosquera J, Gómez E y Peñalosa J. (2012) Construcción colectiva de políticas públicas para el desarrollo humano sostenible. Revista Luna Azul, N 35, ISSN 1909-2474. Universidad de Caldas, Manizales, pp. 116-148 